\title{
Near-field interaction of twisted split-ring resonators
}

\author{
David A. Powell, Kirsty Hannam, Ilya V. Shadrivov, and Yuri S. Kivshar \\ Nonlinear Physics Centre, Research School of Physics and Engineering, Australian National University, Canberra, ACT 0200, Australia
}

(Received 28 January 2011; revised manuscript received 5 April 2011; published 15 June 2011)

\begin{abstract}
We present experimental, numerical, and analytical results for the study of near-field interaction of twisted split-ring resonators, the basic elements of the so-called stereometamaterials. In contrast to previous results, we observe a crossing point in the dispersion curves where the symmetric and antisymmetric modes become degenerate. We introduce a model to describe the interplay between magnetic and electric near-field interactions and demonstrate how this model describes the crossing of the dispersion curves, initially considering lossless identical resonators. Finally, we apply the theory of Morse critical points to demonstrate the competition between losses and differences between the rings in determining whether or not the symmetric and antisymmetric modes cross.
\end{abstract}

DOI: 10.1103/PhysRevB.83.235420

PACS number(s): 81.05.Xj, 78.67.Pt

\section{INTRODUCTION}

Metamaterials created as an array of subwavelength resonant elements can exhibit interesting electromagnetic properties, such as a negative refractive index. ${ }^{1}$ An important building block of metamaterials is the split ring resonator (SRR), ${ }^{2}$ which has a highly dispersive magnetic polarizability that is negative over some frequency band. However, the response of a metamaterial is not simply given by the response of an individual resonator, it depends also on the arrangement of resonators within the system. ${ }^{3}$ Unlike the atoms in natural materials, the near-field patterns of metamaterial elements are quite complex, giving rise to strong interactions between them. This means that the arrangement of resonators in a lattice or superlattice plays an important role in determining the response of the metamaterial. ${ }^{4-10}$ A review of many examples of interaction between such resonant elements can be found in Ref. 11. Characterizing the underlying interaction mechanisms between elements is essential to understanding the overall resonant properties and effective parameters of the material. By controlling the relative arrangement of elements, it is possible to alter the response of the material substantially even if the constituents are fixed.

Recently, H. Liu et al. ${ }^{12}$ analyzed numerically an array consisting of pairs of split ring resonators on the same axis, with the second ring rotated by $90^{\circ}$. The hybridized modes of this system show strong polarization rotation, and have suppressed radiation losses. ${ }^{13}$ Subsequently, N. Liu et al. ${ }^{14}$ investigated a similar system, but considered an arbitrary angle between the two rings. Using numerical analysis, they extracted the eigenmodes of a pair of SRRs operating in the near infrared. They concluded that as the twist angle increases, the resonances converge, undergo an avoided crossing, then diverge again. The reason for this avoided crossing is not clear, since our previous study of a different system of coupled SRRs showed that the hybridized modes can cross. ${ }^{6}$ In Ref. 14 the dispersion curve with an avoided crossing was fitted by a multipole interaction model, however no physical justification for this fitting was given. In particular, it was assumed that the magnetic-interaction constant is invariant with twist angle, however this is inconsistent with the strong variation of current density around the circumference of the ring. This model was subsequently extended to include the polarization-rotation of the scattered radiation. ${ }^{15}$

The purpose of this article is twofold. First, we give a rigorous analysis of the interaction between a pair of coaxial SRRs with one ring rotated, and determine the conditions under which the dispersion curves will undergo a crossing or avoided crossing. We introduce a physically based model for describing the interaction between the rings, and show how this model explains the experimentally and numerically observed dispersion behavior. In Sec. II, we present our experimental and numerical analysis of the dispersion curves. In Sec. III A we show how the dispersion curves can cross, based on an idealized model of a pair of SRRs. Finally, in Sec. III B we consider the influence of losses and nonidentical rings on the dispersion curves. Using the theory of Morse critical points, we will show that competition between losses and differences in the resonant frequencies of the rings will determine whether or not the modes cross.

\section{EXPERIMENTAL RESULTS}

We consider a pair of SRRs with varying twist angle $\theta$ between them shown schematically in Fig. 1(a). First, we perform microwave experiments with a series of rings, with one ring held fixed, and a separate sample created for each rotated ring. The rings used have an inner radius of $3.5 \mathrm{~mm}$, an outer radius of $4 \mathrm{~mm}$, and a gap of $1 \mathrm{~mm}$. They are copper, printed onto 1.6-mm-thick FR4 circuit board, and the rings are $3.6 \mathrm{~mm}$ apart, with the dielectric boards located between the rings. The incoming microwaves are polarized so that the electric field is across the gap of the fixed ring, as shown in Fig. 1(a). To match our experimental data, we preform numerical calculations using CST MICROWAVE STUDIO with the boards having a dielectric constant of 4.6.

Experimental results were measured using a Rohde and Schwarz ZVB network analyzer in a WR-229 rectangular waveguide. We measure the excitation of the rings from the absorption of the system, given by $1-\left|S_{21}\right|^{2}-\left|S_{11}\right|^{2}$, where $S_{21}$ is the transmission coefficient, and $S_{11}$ is the reflection coefficient. A comparison of the experimental and numerical absorption curves for $\theta=90^{\circ}$ is shown in Fig. 1(b). To characterize the dispersion behavior, experiments were 

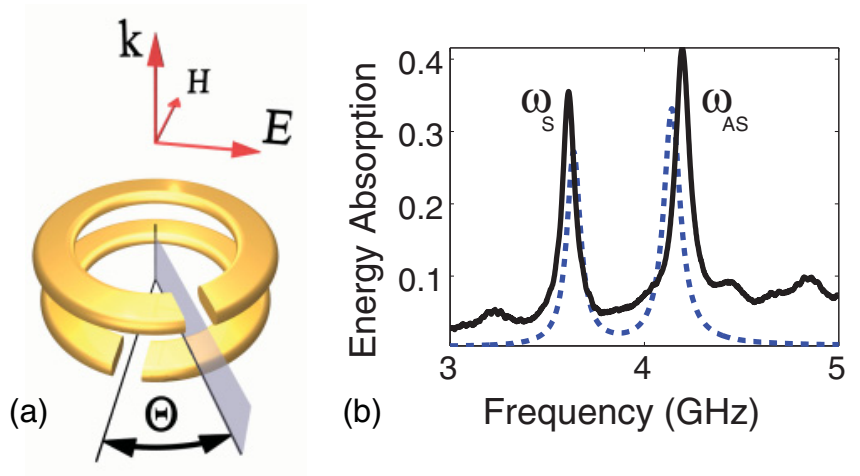

FIG. 1. (Color online) (a) A schematic showing the rings rotated with respect to each other through angle $\theta$, and the polarization of the incoming waves. (b) A comparison of the experimental (solid) and numerical (dashed) absorption for angle $\theta=90^{\circ}$.

performed with $\theta$ varied from $0^{\circ}$ to $180^{\circ}$ in $10^{\circ}$ increments, while numerical results were calculated in $5^{\circ}$ increments. For each angle a Fano function ${ }^{16}$ was fitted to the experimentally and numerically obtained absorption curves, and the resulting resonant frequencies and absorption coefficients are shown in Figs. 2(a) and 2(b), respectively. In all cases the data were well-described by a pair of Fano resonances with a correction for background absorption, and the fitting to the numerical results has a residual error below $5 \times 10^{-3}$.

For $\theta=0^{\circ}$, there are two resonances, $\omega_{S}$ and $\omega_{A S}$, and by inspection of the simulated currents in the rings we
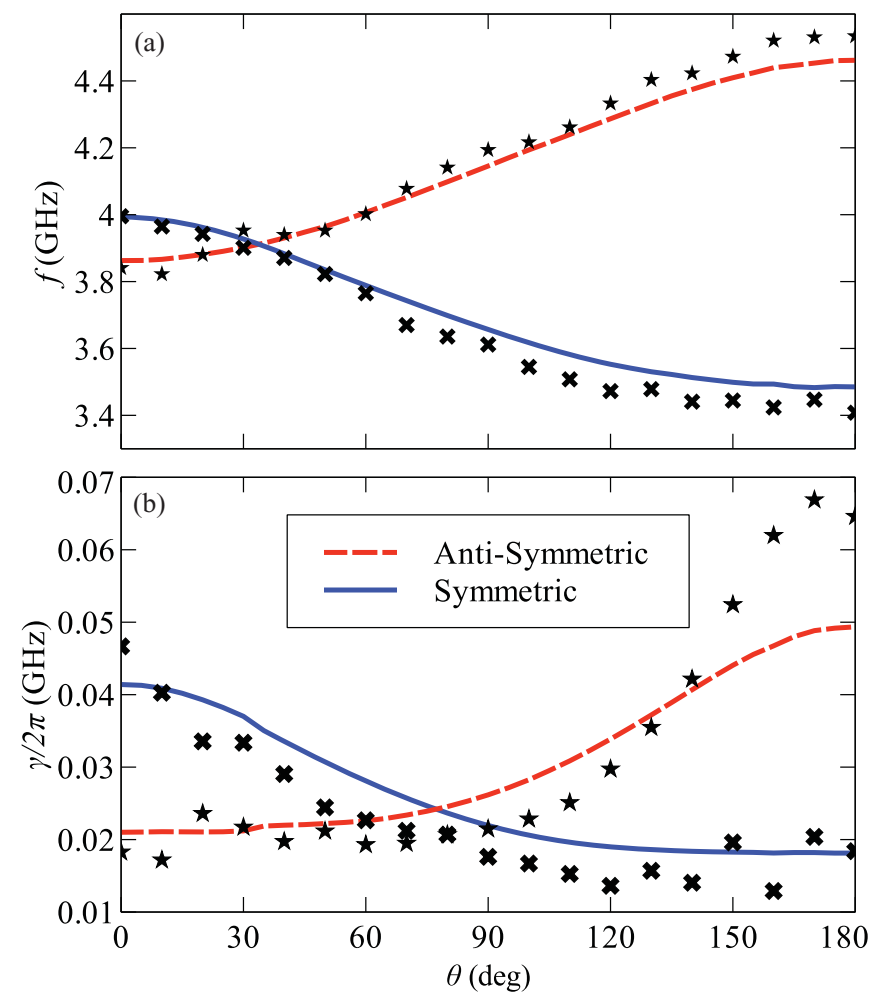

FIG. 2. (Color online) (a) A comparison of the experimental (markers) and numerical (lines) resonant frequencies. (b) Corresponding absorption coefficients, calculated from the resonance linewidths. verify that these correspond to the expected symmetric and antisymmetric modes. As $\theta$ increases, $\omega_{A S}$ increases and $\omega_{S}$ decreases, reaching their maximum and minimum values, respectively, at $\theta=180^{\circ}$. As can be seen in Fig. 2(b) and Fig. 3(a), the two resonant peaks have different widths, which are primarily due to differing radiation losses. The symmetric mode has relatively stronger radiation losses for low angles, since each ring approximates an electric dipole, and a pair of parallel dipoles radiate strongly. As the angle approaches $180^{\circ}$, the dipoles become oppositely directed, thus we have the low radiation efficiency of an electric quadrupole/magnetic dipole-like distribution. ${ }^{17}$ The antisymmetric mode has the charges on one ring of the opposite sign to the other, therefore it changes from an electric-quadrupole- to an electric-dipoletype distribution with increasing angle, and the radiation efficiency increases.

Inspection of Fig. 2(a) shows that the resonances appear to cross at $\theta \approx 34^{\circ}$. Considering only the resonant frequencies, we cannot eliminate the possibility of a narrowly avoided crossing too small to resolve. However the numerically determined loss coefficients $\gamma$ shown in Fig. 2(b) can be directly identified with the corresponding resonant frequencies since they are derived from the same fitted peaks. The absorption coefficients remain clearly distinct in the region of the crossing (confirmed by additional numerical simulations in $0.02^{\circ}$ steps), thus the resonant frequencies are not exchanged and there is a clear indication of a crossing of modes.

In Fig. 3(b), we have plotted the numerical absorption spectrum in the vicinity of the crossing. It has the appearance of only a single peak, however the fitting procedure still identifies two separate resonances, which are also plotted for comparison purposes. In general, the experimental data
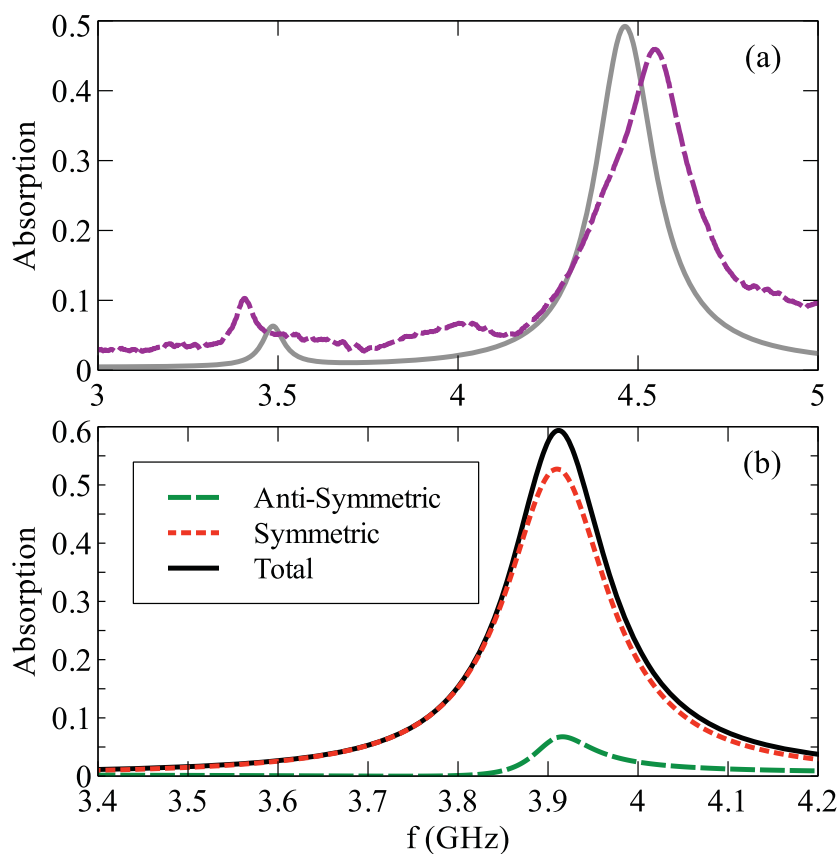

FIG. 3. (Color online) (a) Numerical (solid) and experimental (dashed) absorption curves for $\theta=180^{\circ}$. (b) Numerical absorption curve (solid) for $\theta=34^{\circ}$ at the crossing, showing the two fitted resonances (dashed). 
show good agreement with the numerical data, but with some uncertainties due to fabrication tolerances. We cannot say for certain that there is a crossing of the experimental dispersion curves, only that if an avoided crossing exists then it must be small. Therefore it is necessary to have a theoretical basis for understanding the dispersion curves, which we develop in Sec. III A. Subsequently, in Sec. III B we will include the effects of experimental error into the model, thus the theory can be used to evaluate the reliability of our conclusion from the experimental results.

\section{THEORY OF CROSSING}

\section{A. Identical and lossless rings}

The tuning of the system by rotation can be explained by looking at the interaction between the rings. As the rings are twisted, the magnetic and electric near fields between the two rings change, changing the coupling between them. We first approach this problem using the Lagrangian for a pair of identical and lossless resonators 6,11

$$
\begin{aligned}
\mathcal{L}= & \frac{L}{2}\left(\dot{Q}_{1}^{2}+\dot{Q}_{2}^{2}+2 \kappa_{M} \dot{Q}_{1} \dot{Q}_{2}\right) \\
& -\frac{1}{2 C}\left(Q_{1}^{2}+Q_{2}^{2}+2 \kappa_{E} Q_{1} Q_{2}\right),
\end{aligned}
$$

where $\kappa_{M}$ and $\kappa_{E}$ are the dimensionless magnetic and electric interaction constants, $Q_{1,2}(t)$ are the time-dependent amplitudes of the modes' charge distributions, and the rings have resonant frequency $\omega_{0}=(L C)^{-\frac{1}{2}}$. By substituting Eq. (1) into the Euler-Lagrange equation, the dynamic equations are found to be

$$
\begin{aligned}
& \ddot{Q}_{1}+\omega_{0}^{2} Q_{1}=-\kappa_{M} \ddot{Q}_{2}-\kappa_{E} \omega_{0}^{2} Q_{2}, \\
& \ddot{Q}_{2}+\omega_{0}^{2} Q_{2}=-\kappa_{M} \ddot{Q}_{1}-\kappa_{E} \omega_{0}^{2} Q_{1} .
\end{aligned}
$$

Solving the characteristic equation for this system, gives two resonances: a symmetric $\left(Q_{1}=Q_{2}\right)$, and an antisymmetric $\left(Q_{1}=-Q_{2}\right)$ :

$$
\omega_{S}=\omega_{0} \sqrt{\frac{1+\kappa_{E}}{1+\kappa_{M}}}, \quad \omega_{A S}=\omega_{0} \sqrt{\frac{1-\kappa_{E}}{1-\kappa_{M}}} .
$$

For a pair of rings in a homogeneous dielectric background, the electric and magnetic interaction constants can be determined from the interaction energy between resonators using the method described in Ref. 6. The resulting interaction constants are shown by the markers in Fig. 4(a), along with the following functions, which fit the data very well:

$$
\kappa_{E}=\kappa_{E 1} \cos (\theta), \quad \kappa_{M}=\kappa_{M 0}+\kappa_{M 1} \cos (\theta)
$$

with $\kappa_{E 1}=0.085, \kappa_{M 0}=0.098$, and $\kappa_{M 1}=0.05$. These constants are dictated by the charge separation across the gap of the ring, the current circulating around the ring, and the inhomogeneity of the current distribution around the ring, respectively.

For rings aligned on the same axis, we expect that the magnetic interaction, $\kappa_{M}$, should always be positive, as the intersecting magnetic field from one loop should always be

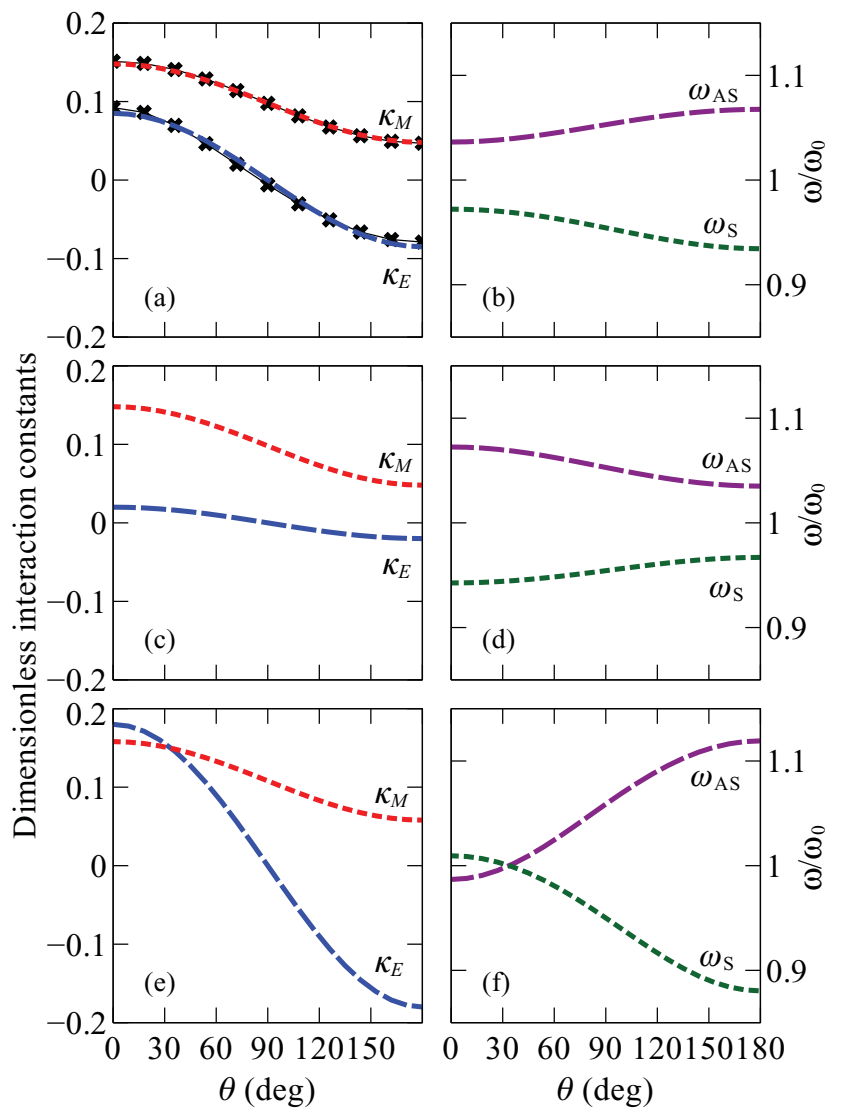

FIG. 4. (Color online) (a) Magnetic $\left(\kappa_{M}\right)$ and electric $\left(\kappa_{E}\right)$ interaction constants calculated for a pair of rings in free space. Dots show exact calculations, lines give the fitted function, and (b) the corresponding resonant frequencies. (c) Interaction where the variation in magnetic coupling dominates and (d) corresponding resonant frequencies. (e) Interaction constants that become equal and (f) corresponding resonant frequencies that cross.

normal to the other loop. In addition, the electric interaction, $\kappa_{E}$, should be positive at $\theta=0^{\circ}$ as the charge distribution has the nature of parallel dipoles. All arrangements of rings on the same axis that we considered behaved in this manner.

In Fig. 4(b), we plot the corresponding frequencies of the symmetric and antisymmetric modes normalized to $\omega_{0}$. As our approach models the response of the resonators in a homogeneous dielectric background, the results are significantly different from those observed experimentally, where the dielectric is inhomogeneous and the effect of waveguide boundaries is also significant. In particular, for this system of perfectly conducting rings, the crossing of resonances cannot be reproduced in a homogeneous background. Therefore, we consider the possible regimes of interaction that may occur under the assumption that the interaction constants will be of the form described in Eq. (4).

The case considered in Figs. 4(a) and 4(b) corresponds to the magnetic interaction always being larger than the electric interaction. This results in increasing splitting of $\omega_{S}$ and $\omega_{A S}$ with increasing twist angle, however, in principle, there is no reason why the splitting cannot decrease. We show such a case in Figs. 4(c) and 4(d), where we have set $\kappa_{E 1}=0.02<$ $\kappa_{M 1}$, such that the inhomogeneity in the current has a stronger 
influence than the dipole-like charge distribution. Despite the different behavior of the frequency splitting curves, there is little qualitative difference between the interaction constants shown in Figs. 4(a) and 4(c).

The only other case allowed in our model of interaction under the aforementioned physical constraints on $\kappa_{M}$ and $\kappa_{E}$ is that $\kappa_{M}>\kappa_{E}$ for $\theta=0^{\circ}$. An example of this is given in Fig. 4(e), where we have set $\kappa_{M 0}=0.108, \kappa_{M 1}=$ 0.05 , and $\kappa_{E 1}=0.18$, such that $\kappa_{E}=\kappa_{M}$ at $\theta \approx 34^{\circ}$. The corresponding resonant frequencies normalized to $\omega_{0}$ are plotted in Fig. 4(f). The parameters have been chosen to closely match the dispersion shown in Fig. 2(a). From this match between the model and the experimental data, we conclude that the inhomogeneous dielectric enhances the electric interaction between the rings but has almost no influence on the magnetic interaction, as expected.

Equations (3) show that the tuning curves arise from a competition between electric and magnetic interaction constants. If the magnetic and electric interaction constants have the same sign, then they will counteract each other. Thus the frequency splitting can be weak even if the near-field interaction is strong. In particular, the model shows that there is no splitting when $\kappa_{M}=\kappa_{E}$, in agreement with the numerical results presented in Fig. 2.

\section{B. Nonidentical and lossy rings}

Up to this point, we have given a theoretical account of interaction, which predicts a crossing of modes, and which is consistent with experimental results and numerical calculations. However, a single ring within the waveguide will have some variation of its resonant frequency as it is rotated, due to interactions with the image currents in the waveguide walls. Similar effects should occur for rings which form part of a larger planar lattice. In addition, there will be further contributions due to fabrication uncertainties. Therefore the pair of twisted rings should be modeled as nonidentical resonators. In addition, the theory developed in Sec. III A has not included the influence of losses, neglecting the strong radiation from the rings into the waveguide modes, as well as ohmic dissipation.

One would expect that differences between the two rings would result in an avoided crossing, following well-known results from coupled-mode theory. ${ }^{18}$ However, such results rely on the Hermitian nature of the system, and are not valid once losses are considered. Therefore, we will investigate this question using the theory of Morse critical points, which has previously been applied to resonators and waveguides. ${ }^{19-21}$ In Ref. 22, an approach was demonstrated which showed the conditions for crossing or anticrossing of modes, and in particular demonstrated that losses can counteract an avoided crossing. We apply this approach to our system and present the key results. For a complete derivation and theoretical background, the reader is referred to Ref. 22.

First, we modify the system of equations (2) by introducing dissipation coefficients $\Gamma_{1,2}$ and a detuning parameter $\delta \omega$ :

$$
\begin{aligned}
& \ddot{Q}_{1}+2 \Gamma_{1} \dot{Q}_{1}+\left(\omega_{0}+\delta \omega\right)^{2} Q_{1}=-\kappa_{M} \ddot{Q}_{2}-\kappa_{E} \omega_{0}^{2} Q_{2}, \\
& \ddot{Q}_{2}+2 \Gamma_{2} \dot{Q}_{2}+\left(\omega_{0}-\delta \omega\right)^{2} Q_{2}=-\kappa_{M} \ddot{Q}_{1}-\kappa_{E} \omega_{0}^{2} Q_{1} .
\end{aligned}
$$

By taking the time dependence of $Q_{1,2}$ as $\exp (j \omega t)$, we arrive at the following dispersion equation:

$$
\begin{aligned}
D(\omega, \theta)= & {\left[\left(\omega_{0}+\delta \omega\right)^{2}+j 2 \Gamma_{1} \omega-\omega^{2}\right]\left[\left(\omega_{0}-\delta \omega\right)^{2}\right.} \\
& \left.+j 2 \Gamma_{2} \omega-\omega^{2}\right]-\left[\kappa_{E}(\theta) \omega_{0}^{2}-\kappa_{M}(\theta) \omega^{2}\right]^{2}=0 .
\end{aligned}
$$

The solutions of this system are not strictly symmetric and antisymmetric, although for small $\delta \omega$ and for angles away from the avoided-crossing they are only slightly perturbed from the original modes. This model includes the differences in radiation losses of the symmetric and antisymmetric modes, which also vary with twist angle. For this example, we take $\kappa_{M}$ and $\kappa_{E}$ as given by Eq. (4), with the same coefficients used to derive Figs. 4(e) and 4(f).

The dispersion curves of the system correspond to the condition $D(\omega, \theta)=0$. In applying the theory of Morse critical points, we study the behavior of the function $D(\omega, \theta)$ in the neighborhood of the crossing/anticrossing, and do not limit ourselves to values of $(\omega, \theta)$ that satisfy the dispersion equation.

The first step is to find the Morse critical point $\left(\omega_{m}, \theta_{m}\right)$ that satisfies $D_{\omega}^{\prime}\left(\omega_{m}, \theta_{m}\right)=D_{\theta}^{\prime}\left(\omega_{m}, \theta_{m}\right)=0$. In the case of a crossing of modes, this will be approximately the point where the dispersion curves cross, in the case of an avoided crossing this will be a saddle-point of $D(\omega, \theta)$. In general, this point must be found numerically, however, if we assume that $\Gamma_{1}=\Gamma_{2}=\Gamma$, we find a sufficiently accurate solution by using perturbation theory to first order in $\Gamma$ :

$$
\begin{gathered}
\omega_{m}=\omega_{m 0}+j \Gamma \\
\omega_{m 0}=\left(\omega_{0}^{2}+\delta \omega^{2}\right)^{\frac{1}{2}} \\
\cos \left(\theta_{m}\right)=\frac{\kappa_{M 0} \omega_{m 0}^{2}}{\kappa_{M 1} \omega_{m 0}^{2}-\kappa_{E 1} \omega_{0}^{2}}+j \Gamma \frac{2 \kappa_{M 0} \kappa_{E 1} \omega_{m 0} \omega_{0}^{2}}{\left(\kappa_{M 1} \omega_{m 0}^{2}-\kappa_{E 1} \omega_{0}^{2}\right)^{2}} .
\end{gathered}
$$

In the vicinity of the Morse point we perform a second-order Taylor expansion of $D(\omega, \theta)$ :

$$
\begin{aligned}
D(\omega, \theta)= & D\left(\omega_{m}, \theta_{m}\right)+D_{\omega \omega}^{\prime \prime}\left(\omega-\omega_{m}\right)^{2} / 2+D_{\omega \theta}^{\prime \prime}(\omega \\
& \left.-\omega_{m}\right)\left(\theta-\theta_{m}\right)+D_{\theta \theta}^{\prime \prime}\left(\theta-\theta_{m}\right)^{2} / 2 .
\end{aligned}
$$

For our system the relevant partial derivatives can be calculated analytically, but the expressions are long and not particularly illuminating. From this local form of the dispersion equation, the solutions are given by

$$
\begin{aligned}
\omega(\theta)= & \omega_{m}-\frac{D_{\omega \theta}^{\prime \prime}}{D_{\omega \omega}^{\prime \prime}}\left(\theta-\theta_{m}\right) \pm\left(D_{\omega \omega}^{\prime \prime}\right)^{-1}\left[\left(D_{\omega \theta}^{\prime \prime}{ }^{2}-D_{\omega \omega}^{\prime \prime} D_{\theta \theta}^{\prime \prime}\right)\right. \\
& \left.\times\left(\theta-\theta_{m}\right)^{2}-2 D_{\theta \theta}^{\prime \prime} D\left(\omega_{m}, \theta_{m}\right)\right]^{\frac{1}{2}}
\end{aligned}
$$

This function has a pair of branch points, where the argument of the square root becomes zero. Upon substitution of the parameters of our system, we find that the branch points occur 

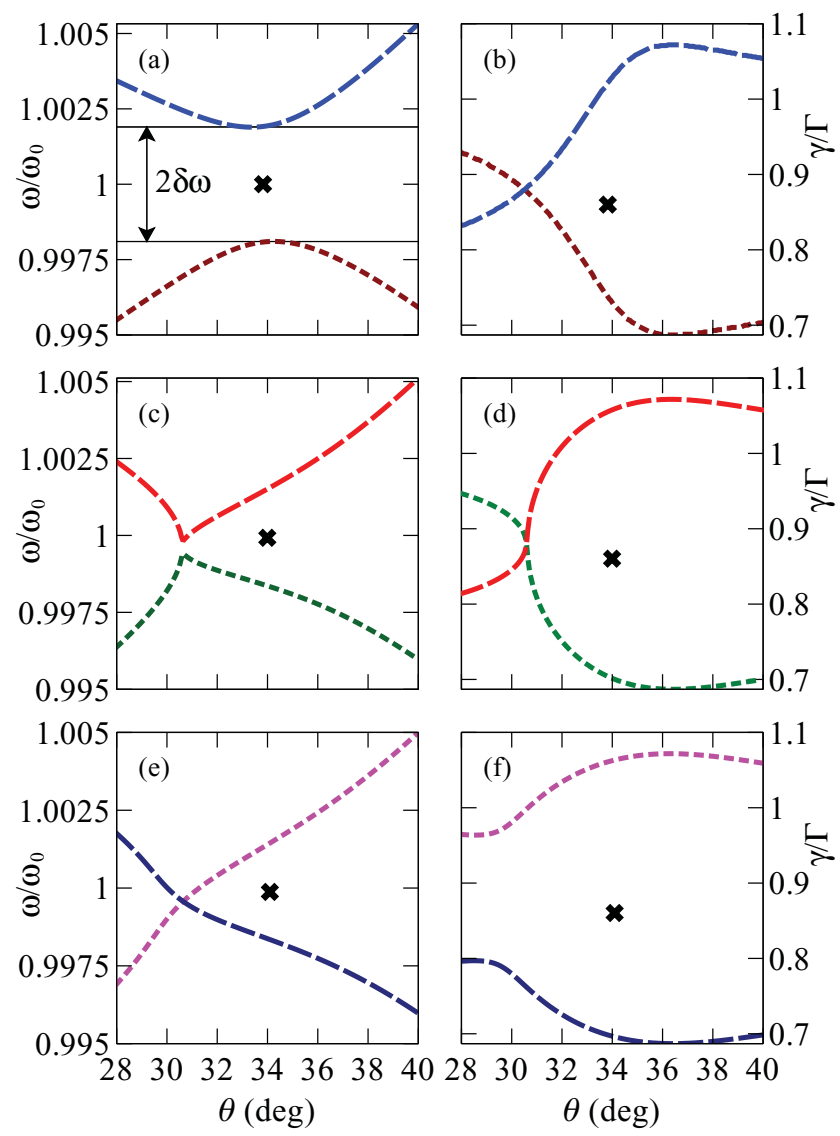

FIG. 5. (Color online) (a) Resonant frequencies and (b) absorption coefficients when $\Gamma=1 \times 10^{-3} \omega_{0}$, (c) Resonant frequencies and (d) absorption coefficients when $\Gamma=7 \times 10^{-3} \omega_{0}$, showing that losses restore the crossing. (e) Resonant frequencies and (f) absorption coefficients when losses are increased to $\Gamma=2 \times$ $10^{-2} \omega_{0}$. The projections of the Morse point are shown by crosses. In all cases $\kappa_{M 0}=0.108, \kappa_{M 1}=0.05$, and $\kappa_{E 1}=0.18$.

at the following values ${ }^{23}$ of $\theta$ :

$\theta_{b 1,2}=\theta_{m} \pm 2 j \delta \omega \omega_{0} \frac{\sqrt{\left(\kappa_{M 0} \kappa_{E 1} \omega_{0}^{2}\right)^{2}-\left(\kappa_{M 1}^{2} \omega_{m 0}^{2}-\kappa_{E 1} \omega_{0}^{2}\right)^{2}}}{\left(\kappa_{M 1} \omega_{m 0}^{2}-\kappa_{E 1} \omega_{0}^{2}\right)^{2}}$.

As shown in Ref. 22, the position of branch points $\theta_{b 1,2}$ on the complex plane determines the behavior of the resonant frequencies of the modes. In brief, if the imaginary parts of the two branch points have different signs, then the modes undergo an anticrossing. However, the modes cross if the imaginary parts of the $\theta_{b 1,2}$ have the same sign. This allows us to analyze the effect of detuning, $\delta \omega$, and losses, $\Gamma$, on the crossinganticrossing behavior of the system. To do this, we numerically analyzed a single ring as it was rotated within the waveguide. By calculating the absorption spectra at $\theta=0^{\circ}$ and $\theta=34^{\circ}$, we find that $2 \delta \omega=3.8 \times 10^{-3} \omega_{0}$, and that $\Gamma_{1}=1.8 \times 10^{-2}$ and $\Gamma_{2}=1.5 \times 10^{-2}$. Therefore, we set $\Gamma_{1}=\Gamma$ and $\Gamma_{2}=$ $0.83 \Gamma$ and evaluate the dispersion behavior as a function of $\Gamma$.

We start with weak losses, when $\Gamma=1 \times 10^{-3} \omega_{0}$. This case is very close to the lossless regime, and the real parts of frequencies are plotted in Fig. 5(a), where we see an avoided crossing, and in Fig. 5(b) we see the imaginary parts, which are exchanged. As we increase losses such that $\Gamma=1.42 \times 10^{-2}$, we see from Fig. 5(c) that the resonant frequencies approach close to each other, but do not cross. The corresponding loss coefficients in Fig. 5(d) still cross over each other. As we further increase losses to $\Gamma=1.8 \times 10^{-2}$ (as obtained from simulations), the dispersion curve changes from an avoided crossing to a crossing, as shown in Fig. 5(e) and 5(f). It is interesting to note that the losses also shift the angle of crossing away from the projection of the Morse point, which is exactly at the crossing in the lossless case.

Further simulations revealed that for $\Gamma=1.8 \times 10^{-2}$, the maximum permissible frequency detuning to maintain a crossing is $2 \delta \omega=4.8 \times 10^{-3}$. This gives a bound on the total difference between the rings, including additional difference due to fabrication error. Such a small fabrication difference should be experimentally achievable, however verifying the experimental resonant frequencies of the individual rings is much more difficult. In our system, to minimize alignment errors, we have fabricated both rings on opposite sides of the board, so we cannot measure the properties of the individual rings. In addition we have a different sample for each angle, thus $\delta \omega$ will vary with angle due to varying fabrication errors. So while we cannot say with certainty that our experimental system has a crossing of modes, we can be confident that any anticrossing would be small, and would be eliminated by improved fabrication tolerances.

From the numerical results in Fig. 2(c) and Fig. 5(d), we see that the decay constants of the hybridized modes are quite different due to different radiation losses. We note that if complete degeneracy of both the frequency and decay constants of the eigenmodes could be achieved, then the coupling between the rings would be completely suppressed. The case shown in Figs. 5(c)-5(d) is very close to this; indeed, there exists a critical value of $\Gamma$ at the transition between the crossing and avoided crossing regimes. In practice, it would be quite difficult to achieve this experimentally, however if such compensation could be realized, this would have important implications for mitigating spatial dispersion.

\section{CONCLUSION}

We have shown in a series of experiments how changing the relative rotation between two rings modifies the electric and magnetic interaction between them, thus tuning the hybridized resonances. Using both numerical simulations and an analytical model, which takes into account both electric and magnetic interactions between the rings, we have shown that there is a crossing where the two resonances coexist, at an angle where the electric and magnetic couplings are equal. We have shown how the waveguide walls and experimental errors can cause the crossing of the dispersion curve to be avoided, however, using the theory of Morse critical points, we have demonstrated that losses can cause the crossing to be restored.

\section{ACKNOWLEDGMENT}

We acknowledge funding from the Australian Research Council. 
*david.a.powell@anu.edu.au

${ }^{1}$ D. R. Smith, W. J. Padilla, D. S. Vier, S. C. Nemat-Nasser, and S. Schultz, Phys. Rev. Lett. 84, 4184 (2000).

${ }^{2}$ J. Pendry, A. Holden, D. Robbins, and W. Stewart, IEEE Trans. Microwave Theory Tech. 47, 2075 (1999).

${ }^{3}$ P. Gay-Balmaz and O. J. F. Martin, J. Appl. Phys. 92, 2929 (2002).

${ }^{4}$ M. Gorkunov, M. Lapine, E. Shamonina, and K. Ringhofer, Eur. Phys. J. B 28, 263 (2002).

${ }^{5}$ M. Lapine, D. Powell, M. Gorkunov, I. Shadrivov, R. Marques, and Y. Kivshar, App. Phys. Lett. 95, 084105 (2009).

${ }^{6}$ D. A. Powell, M. Lapine, M. V. Gorkunov, I. V. Shadrivov, and Y. S. Kivshar, Phys. Rev. B 82, 155128 (2010).

${ }^{7}$ N. Feth, M. König, M. Husnik, K. Stannigel, J. Niegemann, K. Busch, M. Wegener, and S. Linden, Opt. Express 18, 6545 (2010).

${ }^{8}$ I. Sersic, M. Frimmer, E. Verhagen, and A. F. Koenderink, Phys. Rev. Lett. 103, 213902 (2009).

${ }^{9}$ N. Liu and H. Giessen, Opt. Express 16, 21233 (2008).

${ }^{10}$ N. Liu, S. Kaiser, and H. Giessen, Adv. Mater. 20, 4521 (2008).

${ }^{11}$ H. Liu, Y. M. Liu, T. Li, S. M. Wang, S. N. Zhu, and X. Zhang, Phys. Status Solidi B 246, 1397 (2009).
${ }^{12}$ H. Liu, D. A. Genov, D. M. Wu, Y. M. Liu, Z. W. Liu, C. Sun, S. N. Zhu, and X. Zhang, Phys. Rev. B 76, 073101 (2007).

${ }^{13}$ T. Q. Li, H. Liu, T. Li, S. M. Wang, J. X. Cao, Z. H. Zhu, Z. G. Dong, S. N. Zhu, and X. Zhang, Phys. Rev. B 80, 115113 (2009).

${ }^{14}$ N. Liu, H. Liu, S. Zhu, and H. Giessen, Nat. Photon. 3, 157 (2009).

${ }^{15}$ H. Liu, J. X. Cao, S. N. Zhu, N. Liu, R. Ameling, and H. Giessen, Phys. Rev. B 81, 241403 (2010).

${ }^{16}$ A. Miroshnichenko, S. Flach, and Y. S. Kivshar, Rev. Mod. Phys. 82, 2257 (2010).

${ }^{17}$ Y. Zeng, C. Dineen, and J. V. Moloney, Phys. Rev. B 81, 075116 (2010).

${ }^{18}$ H. Haus and W. Huang, Proc. IEEE 79, 1505 (1991).

${ }^{19}$ V. P. Shestopalov, Spectral Theory and Excitation of Open Structures (Naukova Dumka, Kiev, 1987).

${ }^{20}$ V. P. Shestopalov, Electromagnetics 13, 239 (1993).

${ }^{21}$ A. B. Yakovlev and G. Hanson, IEEE Trans. Microwave Theory Tech. 45, 87 (1997).

${ }^{22}$ A. B. Yakovlev and G. Hanson, IEEE Trans. Microwave Theory Tech. 48, 67 (2000).

${ }^{23}$ Strictly speaking, the term inside the square root has some dependence on $\Gamma$, which in turn will cause it to become complex for $\Gamma \neq 0$. However, both effects are negligible in our system. 